\title{
Correction to: Finite Element Solvers for Biot's Poroelasticity Equations in Porous Media
}

\author{
T. Kadeethum ${ }^{1,2}$ (D) S. Lee ${ }^{3}$ H. M. Nick ${ }^{1}$
}

Published online: 26 April 2021

(C) International Association for Mathematical Geosciences 2021

\section{Correction to: Math Geosci (2020) 52:977-1015 https://doi.org/10.1007/s11004-020-09893-y}

The original version of this paper was inadvertently published with an incorrect formula.

The correct formula is

$$
\nabla \cdot \boldsymbol{\sigma}^{\prime}(\boldsymbol{u})-\alpha \nabla \cdot p \boldsymbol{I}=\boldsymbol{f} \quad \text { in } \Omega \times \mathbb{T},
$$

The original article has been corrected. We apologise for any inconvenience caused to our readers.

The original article can be found online at https://doi.org/10.1007/s11004-020-09893-y.

\section{T. Kadeethum}

teekad@dtu.dk; tk658@cornell.edu

S. Lee

lee@math.fsu.edu

H. M. Nick

hamid@dtu.dk

1 Technical University of Denmark, Lyngby, Denmark

2 Cornell University, Ithaca, NY, USA

3 Florida State University, Tallahassee, FL, USA 